compared with participants who had died, for the three psychosocial measures, were: CASP-19 (3.75 and 6.02 respectively, $\mathrm{p}<0.001)$; GHQ-12 (1.24 and 2.12 respectively, $\mathrm{p}<0.001)$; and CES-D (1.53 and 2.44 respectively, $\mathrm{p}<0.001)$. Logistic regression analyses revealed that, after controlling for demographic, health, and lifestyle factors, CASP-19 (odds ratio $(\mathrm{OR})=1.10,95 \%$ CI 1.07 to 1.13), GHO-12 (OR 1.09, 95\% CI 1.05 to 1.12), and CES-D (OR $1.13,95 \%$ CI 1.09 to 1.18 ) all remained significant predictors of death by December 2006. Similar results were obtained using the latent construct.

Conclusion: ELSA offers a unique opportunity to investigate how demographic, health and lifestyle factors influence the ageing process within England. This analysis has established that there is an association between mood and mortality in older adults and suggests that an intervention trial mediating mood is worthwhile.

\section{HEALTH AND DISEASE IN A UK COHORT OF 85-YEAR-OLDS: THE NEWCASTLE 85+ STUDY}

J Collerton, K Davies, C Jagger, A Kingston, M Eccles, 0 James, J Bond, L Robinson, T Von Zglinicki, C Martin-Ruiz, T Kirkwood. Institute for Ageing and Health, Newcastle University, Campus for Ageing and Vitality, Newcastle upon Tyne, UK

\section{doi:10.1136/jech.2009.096727t}

Background: Worldwide, the oldest old are the fastest growing section of the population yet there is little up-to-date information about their health or factors which maintain health and independence. The Newcastle 85+ Study is exploring the spectrum of health within an inception cohort of 85 year-olds and examining health trajectories and outcomes as the cohort ages. Health status at baseline will be presented.

Methods: Members of the 1921 birth cohort were recruited from general practices in Newcastle and North Tyneside, UK during 2006-7. Participation entailed a detailed multi-dimensional health assessment (questionnaires, measurements and fasting blood sample), conducted in the home by a research nurse, together with review of general practice medical records. Undiagnosed disease was estimated by comparing assessment findings with the presence or absence of a recorded diagnosis in the general practice records.

Findings: Of the 1409 individuals contacted, 73.9\% (1041) were recruited; $60.3 \%$ (850) to "face-to-face" assessment plus review of general practice records, $13.3 \%$ (188) to review of general practice records only and $0.2 \%$ (3) to "face-to-face" assessment only. Of the 853 assessed, $62.1 \%$ were female and $10.4 \%$ were living in institutional care. Socio-demographically, the sample was broadly representative of 85-year-olds in Newcastle and North Tyneside and, apart from ethnic diversity, in England and Wales. The most prevalent diseases were hypertension $(57.5 \%)$ and osteoarthritis (51.8\%). With regard to undiagnosed disease, $81.5 \%$ of those with a GDS-15 score suggestive of severe depression had not consulted their GP with depression in the previous year; $53.3 \%$ of those classified as moderately or severely cognitively impaired did not have a diagnosis of dementia and $27.5 \%$ of those with recorded atrial fibrillation were unknown to the GP. A quarter of those without a diagnosis of hypertension had a measured blood pressure in the hypertensive range. Sixty percent reported hearing impairment and over a third visual impairment; $38.3 \%$ reported at least one fall in the previous year; a quarter reported severe or profound urinary incontinence and $8.6 \%$ faecal incontinence. Almost a fifth were fully independent in all 17 activities of daily living and over three-quarters rated their health, compared to others of the same age, as good, very good or excellent with only $3.5 \%$ rating it as poor. Interpretation: The results revealed good overall levels of function and self-rated health in spite of high levels of disease and impairment. Depression, dementia, atrial fibrillation and hypertension appeared to be significantly under-diagnosed.

\section{Parallel session D}

\section{Inequalities II}

\section{CONTEXT OR COMPOSITION? EXPLORING THE ADULT HEALTH AND WELL-BEING OF BRITISH CHILDREN BORN IN DIFFERENT REGIONS}

S Easton. Human Geography, University of Sheffield, Sheffield, UK

doi:10.1136/jech.2009.096727u

Background and Aim: The aim of the research is to explore how multiple social and spatial disadvantage at birth and in early childhood may combine to affect health and well-being in adulthood at age 30 using the British Cohort Study (BCS70). Traditionally researchers examining the geography of poverty or disadvantage have bridged only two disciplines: sociology and geography, or have focused on single outcomes such as mortality or morbidity rates (Dorling, 1997; Tunstall et al, 2007). However this research takes a deeper view of "context", based on Bronfenbrenner's ecological systems theory, where the development of a child depends on the interaction of complex layers in their environment (physical, familial, peers, neighbourhood, wider society). This research therefore crosses several disciplinary boundaries by utilising indicators of early health, cognitive ability and behaviour in the modelling process as well as socioeconomic ones. Some of the variables explored include: birth trauma, breastfeeding, maternal "malaise", antisocial/hyperactive behaviour at age 5 , an index of early adverse events and child health problems.

Adult Outcomes: Three adult outcomes at age 29-30 were modelled taking into account individual/household socioeconomic and spatial variables. These are 1. Satisfaction with Life so far; 2. Self-reported health; 3. Presence of a limiting long-term illness.

Method: Multilevel Modelling: Data were nested by region and the social rating of the neighbourhood at the higher levels and household/individual characteristics at birth and age 5 at the lower level. Spatial level variables such as regional poverty rates at the relevant time (1975) were also included in the models. Multilevel logistic regression and multinomial category response models with MCMC estimation were used to fit the data using MLwiN.

Results: The presence of a limiting long-term illness in adulthood was found to be associated with being female, and low birthweight (linked to socioeconomic position at birth, maternal smoking during pregnancy). The association between low birthweight and having more health problems in childhood was significant. Interaction was found among maternal mental state, living in a poor neighbourhood and child antisocial behaviour. Lower life satisfaction in adulthood was found to be linked to the presence of a congenital abnormality and (socioeconomic position, smoking and) low birthweight, antisocial behaviour in: boys and children from large families, and being poor in a poor neighbourhood. There were interactions between socioeconomic position, cognitive ability and birthweight across the subgroups. Further investigation of Self-reported health at 30 is currently underway.

\section{COMPARISON OF LIFE COURSE SOCIOECONOMIC MODELS FOR CARDIOVASCULAR RISK FACTORS: 1946 BIRTH COHORT}

${ }^{1,2}$ ET Lemelin, ' $\mathrm{G}$ Mishra, ${ }^{1} \mathrm{D}$ Kuh, ${ }^{2} \mathrm{~J}$ Guralnik, ${ }^{1} \mathrm{~S}$ Black, ${ }^{1} \mathrm{R}$ Hardy. ${ }^{1} \mathrm{M} R \mathrm{C}$ Unit for Lifelong Health and Ageing, University College and Royal Free Medical School, London, UK; ' ${ }^{2}$ Laboratory of Epidemiology, Demography, and Biometry Gateway Building, Bethesda, MD, USA

doi:10.1136/jech.2009.096727v

Background: Different approaches have been used to test life course models of socioeconomic position (SEP) in relation to cardiovascular disease (CVD) but have generally only tested one model. 